\title{
Forum
}

\section{Proposal on the Modification of Sailing Calculations}

\author{
T. Hiraiwa
}

(Hokkaido University)

I. INTR ODUCTION. Methods of calculating the course and the distance between two points from a knowledge of their latitudes and longitudes, or calculating the latitude and the longitude of the arrival point from the course and the distance from a known departure point, are called sailings. Middle-latitude sailing and Mercator sailing have been widely used.

In this paper, if the former method is specified, it will include mean middle-latitude sailing and true middle-latitude sailing and these are based on plane sailing and parallel sailing. Mean middle-latitude sailing is an approximate method of computation, therefore it is natural that the calculated result contains some errors.

Accordingly, in this paper, the author attempts to point out the problem of Mercator sailing and the contradictions of the so-called true middle-latitude sailing, to propose modified sailings which avoid their problems, and to describe briefly the other sailings.

From ancient times until today, the methods of calculating a ship's position by dead reckoning have been carried out by treating the Earth as a sphere, or as a sphere in part and a terrestrial spheroid in part. Therefore some problems have arisen. Furthermore, an erroneous method of calculation has been put to practical use by giving priority to convenience, that is, traverse sailing.

2. PLANE SAILING. The present formula is indicated as follows:

$$
\text { D. lat. }=\text { distance } \times \cos \text { (course) }
$$

Here D.lat. is defined as the difference between two latitudes; but the length of a given arc varies with latitude. However, if the distance and the course are the same respectively, D.lat. is calculated as the same figure, irrespective of latitude.

If the radius of the equator is $a$, the eccentricity $e$, and the geographical latitude $\phi$, then the length of the meridian may be expressed as followed:

$$
\mathrm{DLP}=\int_{\phi_{1}}^{\phi_{2}} \frac{a\left(\mathrm{I}-e^{2}\right)}{\left(\mathrm{I}-e^{2} \sin ^{2} \phi\right)^{\frac{3}{2}}} \mathrm{~d} \phi
$$

Williams calls the integrated length from o to $\phi$ in this equation latitude part (LP), and the difference of latitude parts as DLP, and they are measured in units of the length of one minute of arc of the equator. He calculated the length based on Clarke's spheroid (1866) and the extract was indicated in this Journal.' The author made out the table ${ }^{2}$ from $0^{\circ}$ to $90^{\circ}$ every I minute, based on the Bessel's spheroid also.

As LP and DLP are lengths of a meridian, the following equation exists correctly:

$$
\text { DLP }=\text { distance } \times \cos \text { (course) }
$$

Here the unit of DLP is the geographical mile (g.m.); if the distance is given in nautical miles (n.m.) it must be converted into g.m. (In order to do this a conversion table is 
used. $)^{3}$ After calculating DLP by the above-mentioned equation, DLP must be added to LP of Lat. from or subtracted from it, then Lat. in is obtained by the LP table.

3. PARALLEL SAILING. The radius of a parallel of latitude is expressed as $a \cos \phi / \sqrt{ }\left(\mathrm{I}-e^{2} \sin ^{2} \phi\right)$, so the following equation exists :

$$
\text { Dep. }(=\text { distance })=\text { D.Long } \cdot \frac{\cos \phi}{\sqrt{ }\left(\mathrm{r}-e^{2} \sin ^{2} \phi\right)}
$$

If this departure is put as Dep, and the departure which is calculated by the present formula (Dep. $=$ D.Long. $\times \cos \phi$ ) is put as $\operatorname{Dep}_{2}$, the relation that $\operatorname{Dep}_{2} / \operatorname{Dep}_{1}=$ $\sqrt{ }\left(1-e^{2} \sin ^{2} \phi\right)$ exists and $\operatorname{Dep}_{2} \leqq D_{1} p_{1}$ always. The value of $\sqrt{ }\left(1-e^{2} \sin ^{2} \phi\right)$ is larger than 0.9966 . Accordingly if the difference is considered as a ratio, it is close to unity; however, the difference of lengths between $\mathrm{Dep}_{2}$ and Dep $\mathrm{p}_{1}$ can be significant.

4. TRUE MIDDLE-LATITUDE SAILING. If we consider Lat. from as $\phi_{1}$, Lat. in as $\phi_{2}$, course as $\theta$, difference of meridional parts as DMP, the difference of longitudes between the two points is expressed as follows :

$$
\begin{aligned}
\text { D.long. } & =\int_{\phi_{1}}^{\phi_{2}} \frac{a\left(1-e^{2}\right) \tan \theta}{\left(1-e^{2} \sin ^{2} \phi\right) \cos \phi} d \phi \\
& =\frac{\text { Dep. }}{\text { D.lat. } D M P}
\end{aligned}
$$

In middle-latitude sailing, the relation between Dep. and D.Long. is treated as Dep. = D. Long. $\times \cos$ (Mid.lat.), therefore the following relation exists:

So that

$$
\text { Dep. } \times \sec (\text { Mid.lat. })=\text { DMP } \frac{\text { Dep. }}{\text { D.lat. }}
$$

$$
\text { Mid.lat. }=\sec ^{-1}\left(\frac{\text { DMP }}{\text { D.lat. }}\right)
$$

This relation is called true middle latitude. However, there are two questions about the process of derivation. One question concerns the treatment of $\tan \theta$ as Dep./D.lat., and the other is about the relation between Dep. and D.Long. The relation is calculated with the Earth as a sphere, and DMP is calculated with the Earth treated as a terrestrial spheroid. Therefore it is questionable whether the aforesaid relation should be called true middle latitude.

A concrete example of a combination to which a solution cannot be obtained is found when DMP is smaller than D.lat., and the case in which the calculated middle latitude is situated outside both latitudes appears frequently. The limits are indicated in Fig. 1. The portions indicated by dotted lines and broken lines show the combinations where true middle latitudes are situated outside both latitudes.

For example, if Lat $_{1}$ is $0^{\circ}$ a solution does not exist when Lat ${ }_{2}$ is smaller than $11^{\circ} 22^{\prime}$. If Lat $_{2}$ reaches $1_{1}^{\circ} 22^{\prime}, 0^{\circ}$ is obtained as the true middle latitude. And when Lat ${ }_{2}$ grows $^{\circ}$ larger than $11^{\circ} 22^{\prime}$, the mid.lat. increases gradually, and becomes identical with the mean-mid.lat. when $\mathrm{Lat}_{2}$ is $21^{\circ} 35^{\prime}$, and when it is $70^{\circ}$ the true mid.lat. is larger than the mean mid.lat. by more than $10^{\circ}$.

Secondly, in the present formula, that is D.Long. $=$ Dep. $\times \sec$ (Mid.lat.), the following relation is examined:

$$
\text { True mid.lat. }=\sec ^{-1}\left(\frac{\mathrm{DMP}}{\mathrm{DLP}}\right)
$$




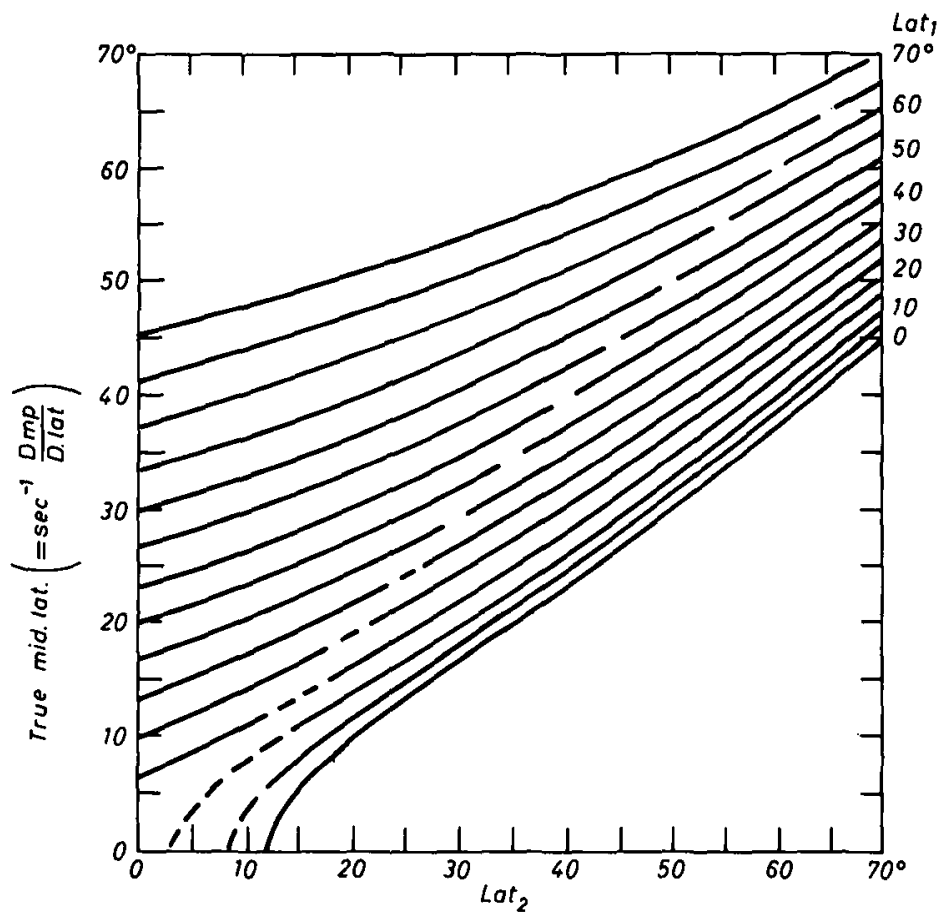

Fig. 1 . True mid.lat. corresponding to any lat ${ }_{1}$ and lat ${ }_{2}$. Here portions indicated by dotted lines and broken lines show the combinations where true middle latitudes are situated out of both latitudes

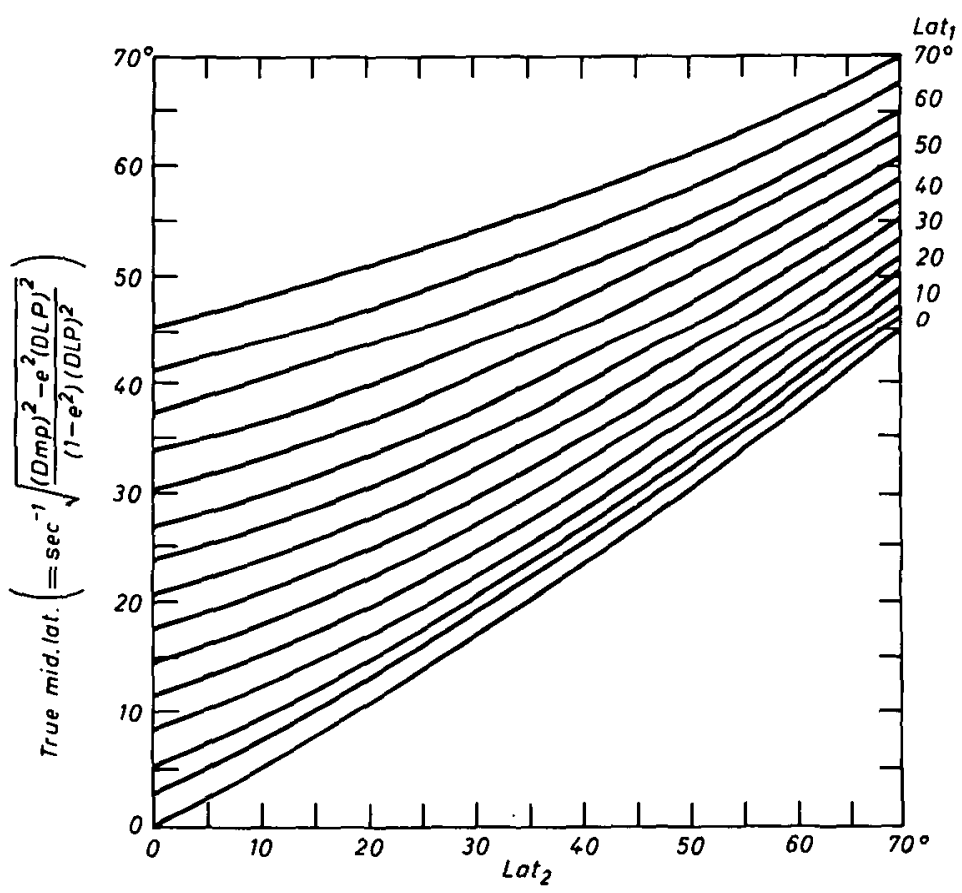

Fig. 2. True middle latitude corresponding to any lat ${ }_{1}$ and lat ${ }_{2}$ 
By employing DLP instead of D.lat. the equation tanCo. = Dep./DLP holds good, based on the length of meridian and Dep. which have been measured in units of the geographical mile. However, the relation between D.long. and Dep. is based on the Earth as a sphere, so the formula can be called a partially modified method as compared with the present sailing.

Furthermore, if the Earth is treated as a terrestrial spheroid, the relation between D.Long. and Dep. is as follows:

therefore

$$
\text { D.long. }=\text { Dep. } \sec \phi \sqrt{ }\left(1-e^{2} \sin ^{2} \phi\right)
$$

$$
\text { True mid.lat. }=\sec ^{-1} \sqrt{\left(\frac{(\mathrm{DMP})^{2}-e^{2}(\mathrm{DLP})^{2}}{\left(1-e^{2}\right)(\mathrm{DLP})^{2}}\right)}
$$

If $e$ is adopted as Bessel's value $(=0.08169683)$, the true middle latitudes are as shown in Fig. 2, and the differences between these values and the former ones which use $\mathrm{sec}^{-1}$ (DMP/DLP) are less than $0.1^{\circ} .4$ The reason is based on the existence of the following relation:

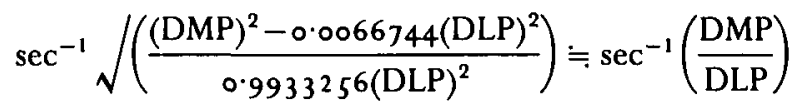

5. TRAVERSE SAILING. Mean middle-latitude sailing is an approximate method from the outset, therefore the calculated result contains some errors naturally. Furthermore, when the method is applied to traverse sailing, another problem arises.

Traverse sailing is the process of calculating an arrival point by adding the differences of latitudes and departures between points for changing course respectively, and calculating the course made good and the distance made good between the departure point and the arrival point when a ship alters its course without sailing directly to the destination.

Even if the method of calculating latitude by addition or subtraction of D.lat. is accepted in practice in spite of the fact that the length of an arc of the meridian varies with the latitude, it is still fundamentally wrong to add or subtract departures. Therefore the D.Long., the course made good and the distance made good calculated according to this method contain errors in all results.

In Fig. 3, let us consider that a ship departs from $A$ and arrives at $B$, and then changes its course towards $\mathrm{C}$. If we use $I^{\circ} \mathrm{N}$. for the latitude of $\mathrm{A}$ and $L^{\circ} \mathrm{E}$. for the longitude of $A$ and $D l_{1}$ for the D.lat. between $A$ and $B$ and Dep, for the departure between $A$ and $B$, and $D l_{2}$ and $D e p_{2}$ for the D.Lat. and departure respectively between $B$ and $C$, the position of $B$ is expressed as :

$$
\left(l+\mathrm{D}_{1}\right) \mathrm{N} .,\left\{L+\mathrm{Dep}_{1} \sec \left(l+\mathrm{D} l_{1} / 2\right)\right\} \mathrm{E} .
$$

and similarly the position of $\mathrm{C}$ is expressed as:

$$
\left(l+\mathrm{D} l_{1}+\mathrm{Dl}_{2}\right) \mathrm{N} .,\left\{L+\mathrm{Dep}_{1} \sec \left(l+\mathrm{Dl}_{1} / 2\right)+\operatorname{Dep}_{2} \sec \left(l+\mathrm{D} l_{1}+\mathrm{D} l_{2} / 2\right)\right\} \mathrm{E} .
$$

Alternatively, the longitude according to traverse sailing may be expressed as:

$$
\left[L+\left(\text { Dep }_{1}+\text { Dep }_{2}\right) \sec \left\{l+\left(\mathrm{Dl}_{1}+\mathrm{Dl}_{2}\right) / 2\right\}\right] \mathrm{E} .
$$

and these two expressions are obviously dissimilar.

As traverse sailing has these intrinsic defects a correct solution cannot be expected. Therefore the author recommends that the sailing should go out of use.

6. MERCATOR SAILING. This method of calculation has been treated as correct, based on the reasoning that it contains no assumption. However, there is a question about D.lat., and accordingly it cannot be said to be a completely satisfactory theoretical sailing. 


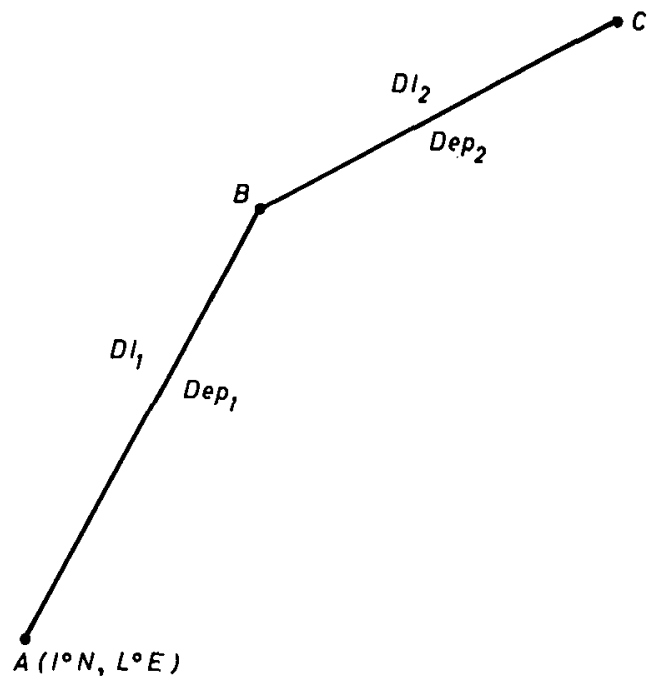

Fig. 3. Relation between departure point (A), point of changed course (B) and arrival point $(C)$

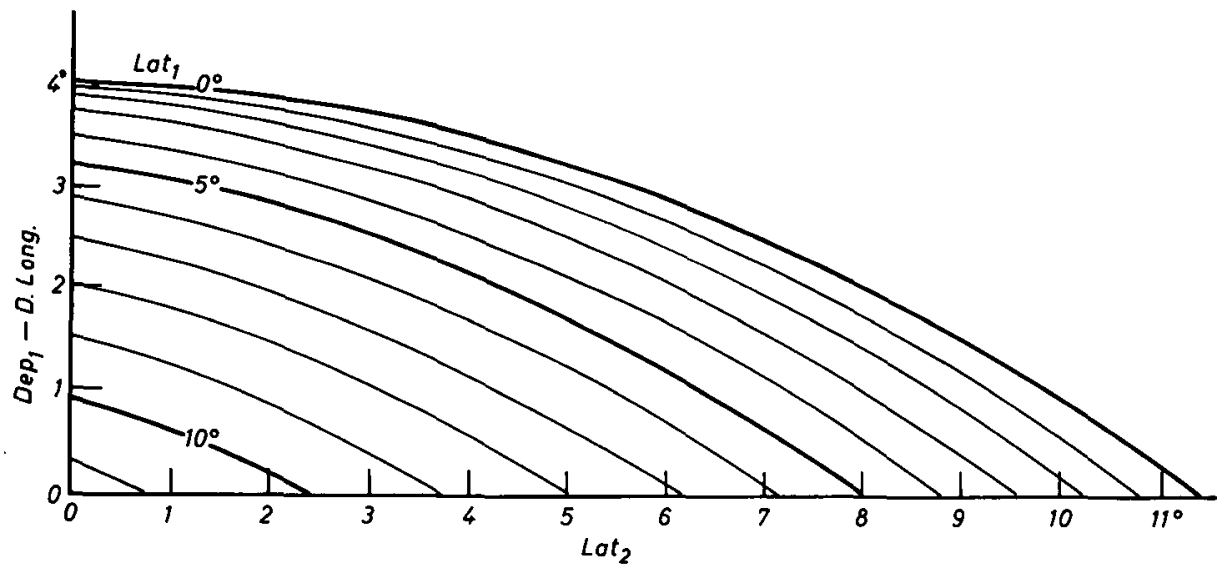

Fig. 4. Difference between the figure of Dep ${ }_{1}$ and of its D.Long. Here D.Long $=10^{\circ}$, Dep $_{1}=$ D.lat $\times$ D. Long. $/$ DMP

The Earth is not a sphere, although as the eccentricity approaches zero, the effect in terms of ratio is small even if the Earth is treated as a sphere in part, and a terrestrial spheroid in part. However, in the numerical calculation of position or distance the result may be significant.

The reason is as follows. Calculations in sailings are normally carried out to one decimal place, but errors which are produced from approximate calculations can exceed this accuracy by a factor of ten or more.

As the distance between two adjacent meridians reduces according to the distance from the equator, it follows that Dep. is always smaller than D.Long. The contrary phenomenon can, however, appear in the Mercator method of calculation and the reason is based on an error of method. So, let us examine the phenomenon by means of a concrete example. 
Example. Calculate (Dep.-D.Long.) when Lat. from is $0^{\circ} \sim 10^{\circ}$, Lat. in is variable, and D.Long. is $10^{\circ}$.

Let us put Dep. calculated by the present formula, that is Dep. $=$ D.lat. $\times$ D. Long./ DMP as Dep ${ }_{1}$, and put Lat. from as Lat ${ }_{1}$ and Lat. in as Lat $_{2}$. The results for (Dep ${ }_{1}$-D.Long.) are shown in Fig. 4. This figure is made up on the condition that D.Long. is $10^{\circ}$, however, if D.Long. is multiplied by an arbitrary constant $n$ (Dep ${ }_{1}$-D.Long.) becomes $n$ times also.

From this figure one may think that Dep, is larger than D.Long., however the result is nothing but a comparison of measurements made in different units. That is to say, the figure shows the results of subtractions which are theoretically inconsistent. The abnormal phenomenon appears where a latitude is lower than $\mathrm{II}^{\circ} \mathbf{2 2}^{\prime}$.

Now if we put Lat ${ }_{1}$ as $\phi_{1}$, Lat ${ }_{2}$ as $\phi_{2}$, then the condition that Dep, equals D.Long. is as follows from the relation D.Lat. = DMP.

$$
\phi_{1}-\phi_{2}=\int_{\phi_{2}}^{\phi_{1}} \frac{a\left(\mathrm{I}-e^{2}\right)}{\left(\mathrm{I}-e^{2} \sin ^{2} \phi\right) \cos \phi} \mathrm{d} \phi
$$

Accordingly $\phi_{1}$ can be solved corresponding to a given $\phi_{2}$.

The above-mentioned matter is a comparison of figures using the present sailing methods. To resolve such a contradiction, Dep. and D.Long. must be measured using the same unit.

The formula when the Earth is treated as a terrestrial spheroid is as follows:

$$
\text { Dep. }=\text { DLP } \times \frac{\text { D. Long. }}{\text { DMP }}
$$

The difference between the current formula and this one is that the units of lengths are standardized as geographical miles by using DLP instead of D.lat. If $\mathrm{Dep}_{2}$ is calculated by the above formula, D.Long. becomes larger than $\mathrm{Dep}_{2}$. And the results for the above-mentioned example are shown in Fig. 5.

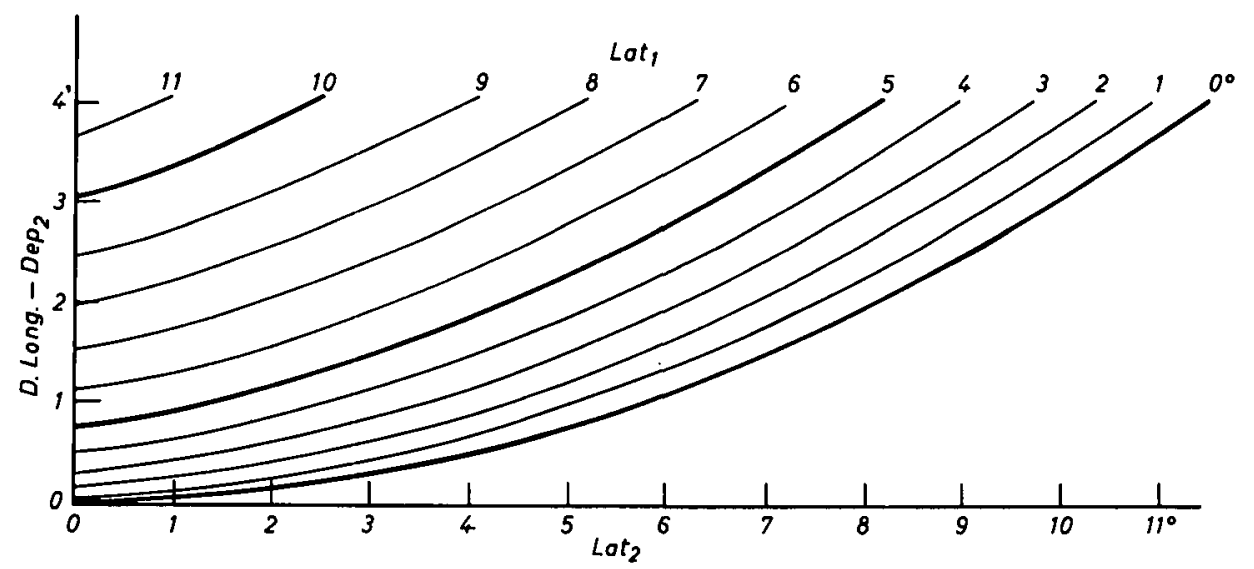

Fig. 5. Difference between D.Long. and Dep. Here D.Long. $=10^{\circ}$, $\mathrm{Dep}_{2}=\mathrm{DLP} \times$ D. Long. $/ \mathrm{DMP}$

7. COMPARISON BETWEEN THE MODIFIED MERCATOR SAILING AND THE MODIFIED TRUE MIDDLE-LATITUDE SAILING. First of all, the present Mercator sailing and the true middle-latitude sailing are examined because both sailings are used 
as identical in certain circles. When true middle latitude is calculated, a sphere and a terrestrial spheroid are combined, and the relation between Dep. and D.Long. is based on a sphere; however, using the following calculation:

$$
\tan \text { Co. }=\frac{\text { Dep. }}{\text { D.lat. }}=\frac{\text { D.Long. } \times \cos (\text { True mid.lat. })}{\text { D.lat. }}=\frac{\text { D.Long. }}{\text { D.lat. }} \times \frac{\text { D.lat. }}{\mathrm{DMP}}=\frac{\text { D.Long. }}{\mathrm{DMP}},
$$

the relation comes to the same result as when the formula for Mercator sailing is used, because $D$.lat. of the denominator and the numerator are eliminated. However, in the calculation of distance D.lat. is used incorrectly in both methods.

By the modified true middle-latitude sailing (tentative name):

therefore

$$
\text { sec }(\text { True mid.lat. })=\sqrt{\left(\frac{(\mathrm{DMP})^{2}-e^{2}(\mathrm{DLP})^{2}}{\left(1-e^{2}\right)(\mathrm{DLP})^{2}}\right)}
$$

so that

$$
\sin (\text { True mid.lat. })=\sqrt{\left(\frac{(\mathrm{DMP})^{2}-(\mathrm{DLP})^{2}}{(\mathrm{DMP})^{2}-e^{2}(\mathrm{DLP})^{2}}\right)}
$$

$$
\tan \text { Co. }=\frac{\text { Dep. }}{\text { DLP }}=\frac{\text { D.Long. } \times \cos (\text { True mid.lat. })}{\text { DLP } \sqrt{ }\left(1-e^{2} \sin ^{2}(\text { True mid.lat. })\right)}=\frac{\text { D.Long. }}{\text { DMP }}
$$

is obtained. Accordingly it is the same as that of Mercator sailing.

In the case of calculating distance, Dist. $=$ DLP $\times \sec$ (course) is employed in both sailings, and the same results are obtained naturally.

8. GREAT CIRCLE SAILING. If the Earth is treated as a spheroid, the shortest distance connecting two points is the length measured along a geodesic line. However, a ship cannot sail on the line, therefore she sails on rhumb lines between some changing course points which are set up previously. Those changing course points cannot be obtained easily, but the eccentricity of the Earth is close to zero, so the Earth may be treated as a sphere and the points calculated on this basis.

The difference between a length of geodesic line and a great circle distance is determined by latitudes and D.Long. of both points. In a former paper $^{5}$ the author made comparisons concerning nine examples.

In this paper, as in the previous examples, he attempts to examine the case when both points are situated on the same parallel of latitude even though it is a special model.

In this case a great circle distance is expressed as follows :

$$
\sigma=\cos ^{-1}\left(\sin ^{2} \phi+\cos ^{2} \phi \cos \right. \text { D. Long. }
$$

On the other hand, there is no easy method of calculating a length of geodesic line directly, so the correction method by Andoyer ${ }^{6}$ and Lambert ${ }^{7}$ is often used. By this method,

$$
\text { correction distance }=\frac{E}{2}\left(1-\sqrt{ }\left(1-e^{2}\right)\right)\left(\frac{3 \sin \sigma-\sigma}{\cos ^{2} \frac{1}{2} \sigma}\right) \sin ^{2} \phi
$$

where

$\sigma:$ great circle distance (radian);

$\phi:$ geographical latitudes of both points;

$E$ : equatorial radius;

$e$ : eccentricity of the Earth.

Now if Bessel's values are employed

correction distance (g.m.) $=5.745^{8}(3 \sin \sigma-\sigma) \sec ^{2} \frac{1}{2} \sigma \sin ^{2} \phi$ 


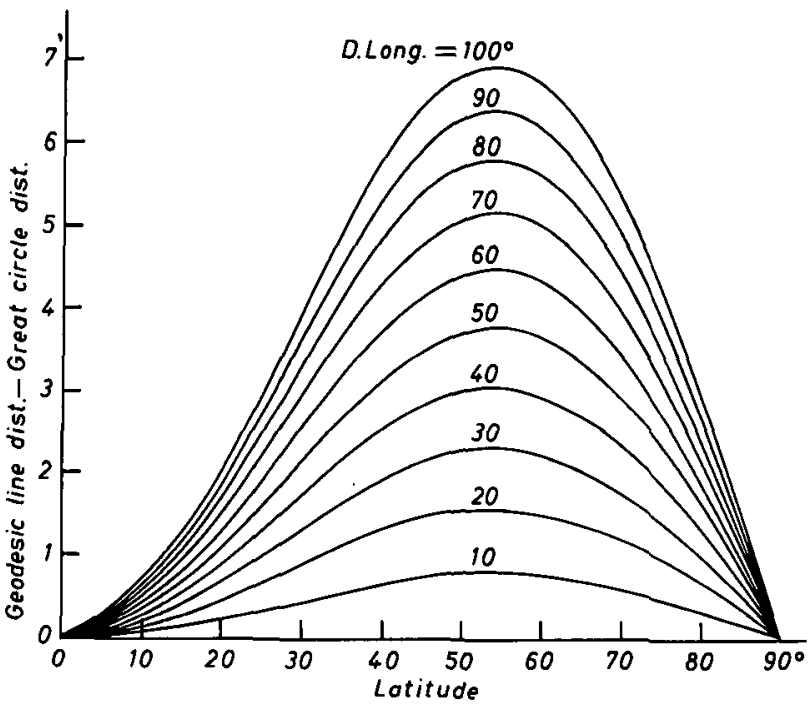

Fig. 6. Difference between geodesic-line distance and great circle distance when D. Long. are $10^{\circ} \sim 100^{\circ}$ and latitudes of two points are alike

is produced. Thus if $\phi$ and D.Long. are given, $\sigma$ is calculated so the correction distance is produced. The results are shown in Fig. 6 in the case of D. Long. of $100^{\circ}$ and below. From this figure, correction distances are seen to be small in low latitudes and high latitudes. However the ratios between correction distances and great circle distances are small in low latitudes and increase gradually with the increase of latitude. The upward tendency and the reason that the ratios are not affected by D.Long. are indicated in the following equation:

$$
\text { correction distance (g.m.) }=\frac{\text { I } 14916(3 \sin \sigma-\sigma)}{1+\cos \sigma} \sin ^{2} \phi
$$

if $\sin \sigma$ and $\cos \sigma$ are expanded and high-order terms are neglected:

$$
\begin{aligned}
\text { correction distance (g.m.) } & =\frac{\mathrm{J} 1 \cdot 49 \mathrm{I} 6 \sigma\left(48-\mathrm{J} 2 \sigma^{2}+0.6 \sigma^{4}\right) \sin ^{2} \phi}{48-\mathrm{I} 2 \sigma^{2}+\sigma^{4}} \\
& \fallingdotseq 1 \mathrm{I} \cdot 4916 \sigma \sin ^{2} \phi
\end{aligned}
$$

Compared with this, the great circle distance (g.m.) $=E \sigma=3437 \cdot 7468 \sigma$, therefore

$$
\frac{\text { correction distance }}{\text { great circle distance }} \fallingdotseq 0.003343 \sin ^{2} \phi
$$

is derived, in which $\sigma$ disappears.

As correction distances are plus in these cases, one may think that a geodesic line distance is longer than a great circle distance. However, the result is nothing but a comparison of the shortest distances on two different curved surfaces. A great circle distance is a hypothetical length founded on a false assumption; it is a non-existent length on the real Earth.

Secondly, an actual sailing distance is the sum total of rhumb-line distances connecting each pair of changing course points. On this basis, the distances calculated by the present formula, that is Dist. $=$ D. lat. $\times \sec$ Co., and the modified formula, Dist. $=$ DLP $\times$ 
Table I. Distances between two points When both points are situated on the same PARALlEL OF LATITUDE AND D.LONG. IS $100^{\circ}$

\begin{tabular}{ccccc}
\hline & \multicolumn{4}{c}{ Distance } \\
\cline { 2 - 5 } Latitude $\left(^{\circ}\right)$ & $\mathrm{D}_{1}$ & \multicolumn{1}{c}{$\mathrm{D}_{2}$} & $\mathrm{D}_{3}$ & \multicolumn{1}{c}{$\mathrm{D}_{4}$} \\
\hline 10 & 5876.8 & 5877.3 & 5913.7 & 5877.5 \\
20 & 5525.0 & 5526.9 & 5553.5 & 5527.3 \\
30 & 4987.3 & 4991.2 & 5006.6 & 4992.0 \\
40 & 4311.8 & 4317.6 & 4323.3 & 4318.3 \\
50 & 3539.8 & 3546.7 & 3546.0 & 3547.5 \\
60 & 2702.5 & 2709.3 & 2705.4 & 2710.0 \\
70 & 1822.7 & 1828.1 & 1823.8 & 1828.5 \\
80 & 917.3 & 920.3 & 917.7 & 920.6 \\
\hline
\end{tabular}

Here $D_{1}$ : great circle distance (g.m.); $D_{2}$ : geodesic-line distance (g.m.); $D_{3}$ : rhumb-line distance $(=\Sigma(\mathrm{D}$.lat. $\times \sec \mathrm{Co})).(\mathrm{min})$ when the changing course points are set up each $5^{\circ}$ of D.Long; $\mathrm{D}_{4}$ : rhumb-line distance $(=\Sigma(\mathrm{DLP} \times \sec \mathrm{Co})).(\mathrm{g} . \mathrm{m}$.$) when the changing course points$ are set up each $5^{\circ}$ of D.Long.

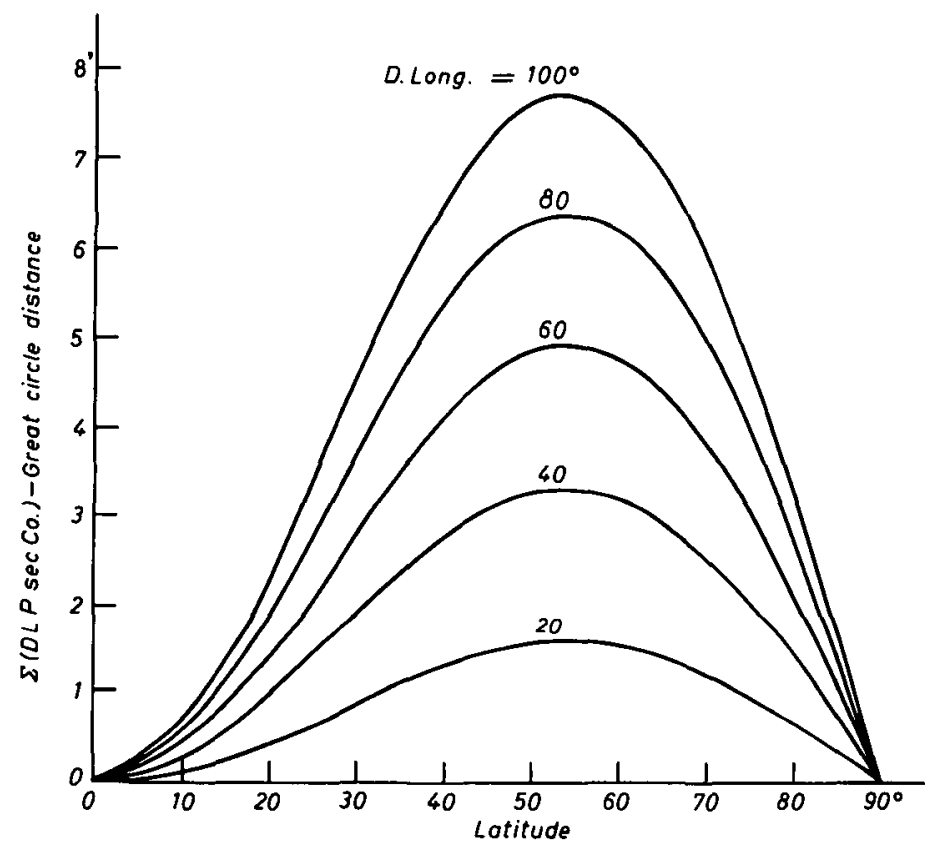

Fig. 7. [ $\Sigma$ (DLP sec Co.)-great circle distance] and D.Long. corresponding to each latitude

sec Co., are examined. As an example, great circle distances, geodesic-line distances, and rhumb-line distances when the changing course points are set up each $5^{\circ}$ of D.Long., are compared for the conditions that $\phi=10^{\circ}, 20^{\circ}, \ldots, 80^{\circ}$, D. Long. $=100^{\circ}$. The results are indicated in Table 1 .

From this table, $D_{4}-D_{1}$ and $D_{2}-D_{1}$ show a similar tendency and value. On the other hand, $D_{3}-D_{1}$ shows an obviously very different configuration. To compare these differences with Fig. 6, the author has examined the cases when D.Long. is $20^{\circ}, 40^{\circ}$, 


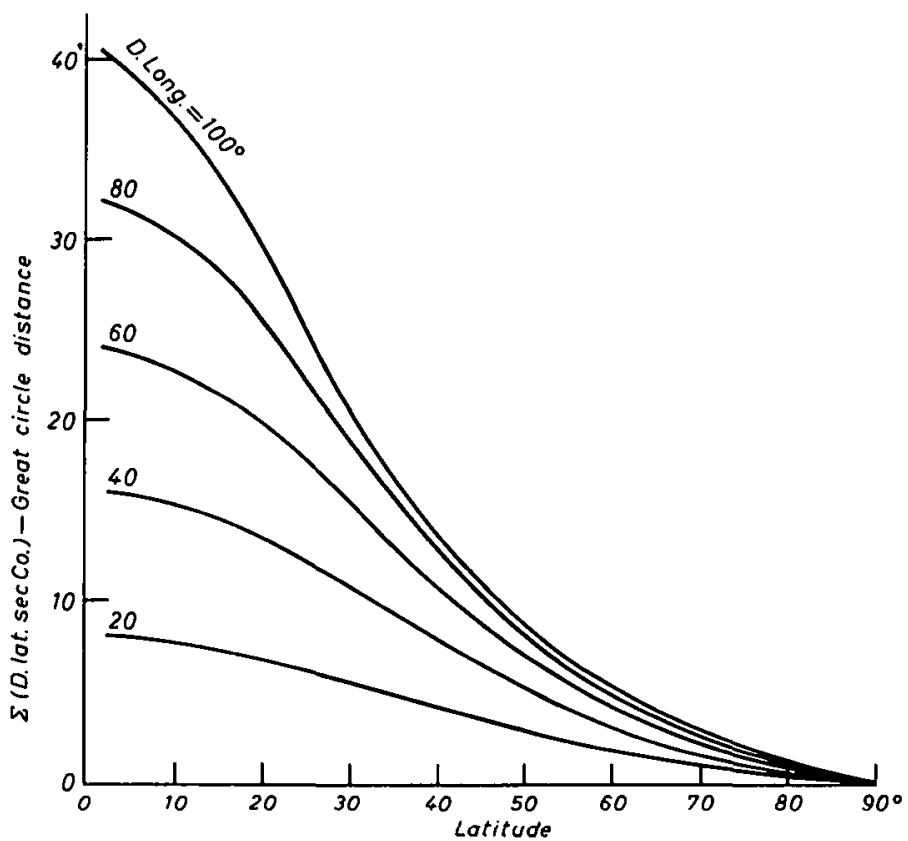

Fig. 8. Relation between [ $\Sigma(D$.lat. sec Co.)-great circle distance] and D.Long. corresponding to each latitude

$60^{\circ}, 80^{\circ}, 100^{\circ}$, and has indicated the results in Fig. 7 and Fig. 8. $D_{3}$ in Table $I$ and the rhumb-line distances in Fig. 8 are calculated by using D.lat., so the meaning of a unit (minute of arc) is ambiguous. Thus comparison with lengths having units of g.m. is not correct theoretically; however, as the method has been used from ancient times until today, the author has compared those figures for reference.

On this basis, the actual sailing distances calculated by $\Sigma($ DLP $\times$ sec Co. $)$ agree with geodesic-line distances within one g.m., therefore the significance of calculating distances down to a tenth of a mile for each section is justified. In other words, the use of the partly modified great circle sailing to calculate changing course points on the sphere and to calculate the length between two changing course points on the terrestrial spheroid is effective in practice because the sum of the loxodromic distances is close to the geodesic-line distances.

9. CONCLUSION. The author has described how the present sailings are not correct theoretically, and that the cause is founded on the fact that the Earth is treated as a sphere or a terrestrial spheroid without coordination. According to the author's analysis, figures which have different units are compared carelessly, or a variable is treated as a constant, and some theoretically absurd sailings have been used for convenience.

To resolve these contradictions, it is necessary to unify the scales used for different quantities and to employ a correct method. As the unit of length it is convenient to use g.m. for the reason that latitude parts, meridional parts and Dep. in parallel sailing are indicated by the unit of g.m. It would be convenient if a ship's speed was expressed in terms of the unit of g.m., so that it was the same unit as for distance and the trouble of converting n.m. into g.m. would be avoided. (On the other hand, some quarters have suggested that it would be better to unify all lengths in terms of the unit of n.m. This is certainly an alternative method, but the process is slightly more troublesome in comparison with the former one.) 
In conclusion, the modified Mercator sailing (tentative name) does not contain any unwarranted assumption and it is easy to apply. Or again the modified true middle-latitude sailing (tentative name) can get the same results, although the calculation is more troublesome. As to great circle sailing, the aforesaid partly modified method is effective in practice.

Finally, the author wishes to add that numerical calculations in sailing are usually carried out down to one decimal place, but if the formulas have some defects the results are not reliable to this order of accuracy.

\section{REFEREN CES}

1 Williams, R. (1981). A table of latitude parts. This Journal, 34, 247.

2 Hiraiwa, T. $\left(\mathrm{r}^{2} 8_{3}\right)$. On the table of latitude parts. Navig. (Tokyo), 75, 61 .

${ }^{3}$ Hiraiwa, T. (1983). Some problems on sailings. Ibid., 76, 1 .

4 Hiraiwa, T. (1983). Modifications of sailings. Ibid., 77, 7 .

${ }^{5}$ Hiraiwa, T. (1984). Modifications of sailings - II. Partial modification of great circle sailing. Ibid., 81, 8.

6 Andoyer, H. (1932). Formule donnant la longueur de la géodésique joignant 2 points de l'ellipsoïde donnés par leurs coordonnées géographiques. Bull. Géodésique, 34, 77.

7 Lambert, W. D. (1942). The distance between two widely separated points on the surface of the earth. J. Washington Acad. Sci., 32, 125. 